\title{
Developing Green Super Rice Varieties with High Nutrient Use Efficiency by Phenotypic Selection Under Varied Nutrient Conditions
}

\author{
Zilhas Ahmed Jewel 1, , Jauhar Ali 1,*, , Yunlong Pang 1,2,3, Anumalla Mahender 1, Bart Acero ${ }^{\text {, }}$ \\ Jose Hernandez ${ }^{4}$, Jianlong $\mathrm{Xu}^{2}$, and Zhikang $\mathrm{Li}^{2, *}$ \\ 1 Rice Breeding Platform, International Rice Research Institute (IRRI), Los Baños, Laguna 4031, Philippines; \\ jeweluplb@gmail.com (Z.A.J.); m.anumalla@irri.org (A.M.); bartzjr16@gmail.com (B.A.) \\ 2 Institute of Crop Sciences, Chinese Academy of Agricultural Science, Beijing 100081, China; \\ zhkli1953@126.com (Z.L) \\ 3 State Key Laboratory of Crop Biology, College of Agronomy, Shandong Agricultural University, Taian \\ 271018, China; y.pang@sdau.edu.cn \\ 4 Institute of Crop Science, College of Agriculture and Food Science, University of the Philippines Los Baños, \\ Laguna 4031, Philippines; joehernandez56@gmail.com \\ * Correspondence: J.Ali@irri.org; Tel.: +63-2580-5600 (ext. 2541); and zhkli1953@126.com \\ $\S$ These authors contributed equally to this work.
}

\begin{abstract}
To develop green super rice varieties with high and stable yields under the rainfed conditions and improved nutrient use efficiency (NuUE), a modified backcross (BC) breeding approach was adopted using a high yielding and widely adaptable Xian variety, WTR1, as the recipient and a Geng variety, $\mathrm{HAN}$, as the donor. Starting from the $\mathrm{BC}_{1} \mathrm{~F}_{2}$ generation, the $\mathrm{BC}$ population had gone through one generation of selection under the IG, LI and RF conditions, followed by consecutive four generations of screening and selection for high GY under six different nutrient conditions, leading to the development of $230 \mathrm{BC}_{1} \mathrm{~F}_{6}$ introgression lines (ILs). The final evaluation of the 230 ILs under the six nutrient conditions identified many ILs with improved yields under various combinations of nutrient deficient conditions, including 12 promising lines that had significantly improved NuUE under two or more nutrient deficiency conditions. Our results demonstrated an efficient inter-subspecific BC breeding procedure with first round selection under the rainfed-drought condition followed by four generations of progeny testing for yield performances under six different nutrient conditions. The promising ILs were studied under replicated yield trials under $75 \mathrm{~N}$ and -NPK conditions for developing high yield rice varieties with improved NuUE. Our results indicated that NuUE in rice was controlled by complex genetic and physiological mechanisms and the developed ILs provided useful materials for genetic and molecular dissection of NuUE in rice.
\end{abstract}

Keywords: nutrient use efficiency, grain yield, nitrogen, phosphorus, potassium, green super rice, BC breeding. 


\section{Introduction}

Rice remains the most important cereal crop and principal staple food in developing countries, particularly in Asia [1]. The global rice production has to increase by about $25 \%$ by 2025 to keep the base year 2001, with a target of 732.5MT of rice produced each year. The world rice production during the Green Revolution (GR) and post-GR was more than doubled [2]. This increased rice production was primarily achieved through the development and wide adoption of rice varieties that were highly responsive to high inputs (chemical fertilizers, pesticides, and ample irrigation water) [3]. These breeding objectives predominated over the last three to four decades but now lie exhausted amidst our hope to raise productivity per se sustainably. However, the crop's efficiency in harnessing applied inputs led to yields approaching a theoretical limit [4, 5]. Single-year fertilizer N recovery efficiencies in researcher-managed experimental plots averaged $46 \%$ for rice crop [6]. However, the experimental plots do not accurately reflect the recovery efficiencies available on-farm. Scale in farming operations differences and management practices such as tillage, seeding, weed and pest control, irrigation, and harvesting usually result in lower nutrient use efficiency. Nitrogen recovery in crops grown by farmers rarely exceeds $50 \%$ and is often much lower. The average nitrogen recovery efficiency in farmer's fields ranges from $20 \%$ to $30 \%$ under the rainfed conditions and from $30 \%$ to $40 \%$ under the irrigated conditions [7]. In most of the time, only half of the applied nutrients used by rice plants and a significant part of nitrogen in the fields lost through volatilization, leaching, de-nitrification, and soil erosion $[8,9]$.

Over the years, rice varieties have not been intentionally improved to maximize nutrient absorption. It is, therefore, necessary to breed rice cultivars with improved nutrient use efficiency (NuUE). Recently, green super rice (GSR), defined as rice varieties that can produce high and stable yields under fewer inputs (water, nutrients, and pesticides) and adverse conditions, was a new concept for enhancing rice NuUE and achieving sustainable rice production through breeding [3, 10]. Breeding varieties with higher NuUE are essential not only to improve yield and reduce production costs but also to avoid environmental pollution and maintain the sustainability of cropping systems [11]. In the light of high energy costs and increasingly fluctuating resources, future agricultural systems need to be more productive and efficient, including for fertilizer and water. Also, improved NuUE is an essential prerequisite for the extending the crop production into marginal soils with lower nutrient availability such as nitrogen, phosphorus, and potassium [11]. Thus, developing rice varieties with high grain yield under low-nutrient conditions has become a breeding priority [12]. Cultivars with higher NuUE, coupled with best management practices, will contribute to sustainable agricultural systems protecting and promoting soil, water, and air quality [13]. Unfortunately, only a few systematic breeding efforts until now have been intended in this respect. In these cases, breeding populations were screened under varying rates of $\mathrm{N}$ and $\mathrm{P}$ fertilizers for identification of NuUE varieties [14-18].

Here, we report an effort for developing GSR varieties with improved NuUE using a unique breeding approach through selecting introgression lines (ILs) with higher NuUE in a BC breeding program, which is potentially going to be widely adopted for improving NuUE of rice varieties in future.

\section{Materials and Methods}

\subsection{Plant materials}

A $\mathrm{BC}_{1} \mathrm{~F}_{2}$ population developed from a cross between an elite Xian (indica) variety, Weed Tolerant Rice1 (WTR1, as the recipient) and a Chinese Geng (japonica), Hao-An-Nong (HAN as the donor) [19]. WTR1 is a high yielding from south China with wide adaptability across subtropical and tropical areas of Asia. WTR1 was crossed with HAN in the 2010 dry season (DS) and the F1 plants 
were backcrossed to WTR1 once in the 2010 wet season (WS). In the 2011 DS, seeds from more than 25 segregating $\mathrm{BC}_{1} \mathrm{~F}_{1}$ plants were bulk harvested without selection to form a single $\mathrm{BC}_{1} \mathrm{~F}_{2}$ population.

\subsection{Selection schemes for improving NuUE}

Figure 1 shows the phenotypic selection schemes of the $\mathrm{BC}_{1} \mathrm{~F}_{2}$ bulk population to develop ILs with improved NuUE at the International Rice Research Institute (IRRI at $14^{\circ} 13^{\prime} \mathrm{N}$ and $121^{\circ} 15^{\prime} \mathrm{E}$, at an elevation of $21 \mathrm{~m}$ above mean sea level), Philippines) during the three wet seasons of 2011-2013 and two dry seasons of 2012 and 2013, respectively. The first round of single-plant selection for higher grain yield was practiced on the $\mathrm{BC}_{1} \mathrm{~F}_{2}$ bulk population grown under irrigated conditions (IG), low-input (LI) and rainfed (RF) situations in 2011WS, resulting in 46 selected $\mathrm{BC}_{1} \mathrm{~F}_{2}$ plants. Then, in the following seasons, four rounds of line-based selection were carried out from progeny testing under six different nutrient input conditions, including the NPK, 75N, -N, -P, -NP, and -NPK conditions. The NPK condition was the condition with total applied NPK fertilizers of 160-50-50 $\mathrm{kg} / \mathrm{ha}^{-1}$ in the DS and $90-30-30 \mathrm{~kg} / \mathrm{ha}^{-1}$ in the WS. The $75 \mathrm{~N},-\mathrm{N},-\mathrm{P},-\mathrm{NP}$, and -NPK conditions indicated that $75 \%$ of nitrogen, the absence of $\mathrm{N}$, the absence of $\mathrm{P}$, the absence of both $\mathrm{N}$ and $\mathrm{P}$, and finally absence of NPK were used, respectively, compared with the normal NPK condition. In each season, seeds of each selected line and parents were sown on the seedling nursery, and 2420 -day old seedlings of each line were transplanted into a two-row plot at a spacing of $20.0 \mathrm{~cm} \times 15.0 \mathrm{~cm}$ with one seedling per hill. The selection was practiced by selecting the best 1-3 plants from the best yield performing lines.

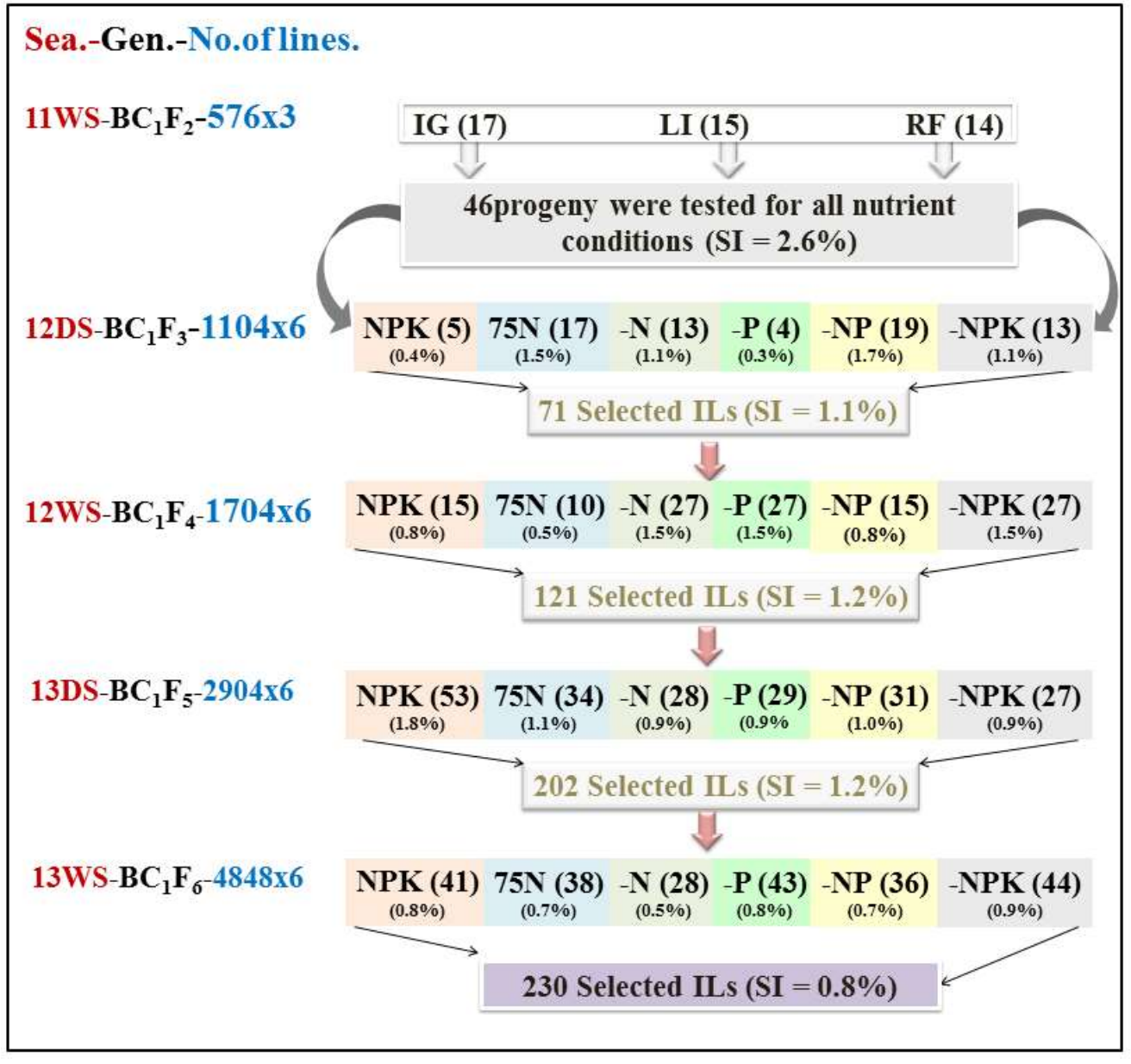

Figure 1. Phenotypic selection schemes of the BC1F2 bulk populations to develop 230 nutrient use efficient ILs [WTR1 = Weed Tolerant Rice 1 (recipient); HAN = Hao-an-nong (the donor); IG = the 
irrigated conditions; LI = the low-input conditions; RF = the rainfed conditions; ILs = introgression lines; SI = selection intensity; Sea = season; Gen = generation; No. of lines =number of lines and grown under six nutrient conditions; $\mathbf{D S}=$ dry season and $\mathbf{W S}=$ wet season]

Phenotyping of grain yield attributed traits of NuUE-ILs: The final evaluation of the $230 \mathrm{BC}_{1} \mathrm{~F}_{7}$ ILs for NuUE was conducted in replicated field experiments under the same six levels of the nutrient input: NPK, 75N, -N, -P, -NP, and -NPK conditions at IRRI in the 2013DS. Seeds of the 230 ILs and parents were sown on the seedling nursery, and 20-day old seedlings of each line were transplanted into a two-row plot with 24 plants or hills at a spacing of $20.0 \mathrm{~cm} \times 15.0 \mathrm{~cm}$, with one seedling per hill and two replications for each line. Weeds were controlled by using herbicides and hand pulling methods. At the maturity, three plants were diagonally sampled in the middle row of each plot for the phenotypic evaluation of 13 agro-morphological and grain yield traits. These traits included plant height $(\mathrm{PH}, \mathrm{cm})$, flag leaf length (FLL, cm), flag leaf width (FLW, mm), flag leaf area (FLA, $\mathrm{cm} 2)$, tiller number $(\mathrm{TN})$, heading date (HD), spikelet number per panicle (SN), panicle length (PL, $\mathrm{cm}$ ), filled grain number per panicle (FGN), spikelet fertility (SF), 1000-grain weight (TGW), biomass $(\mathrm{BM}, \mathrm{g})$, and grain yield per plant $(\mathrm{GY}, \mathrm{g})$.

Statistical data analysis: Data of the measured morphological, agronomical and yield traits were analyzed using Microsoft Excel software. Analysis of variance (ANOVA) was used to determine the significant differences among different treatments $(\mathrm{T})$ of $(\mathrm{NPK}, 75 \mathrm{~N},-\mathrm{N},-\mathrm{P},-\mathrm{NP}$, and -NPK) nutrient input, among lines (G), and GxT interactions using SAS program 9.1 (SAS Institute Inc, Cary Nc). Duncan Multiple Range Tests (DMRT) was used to compare the differences between the mean values of the treatments and genotypes with the help of R software [20].

\section{Results}

\subsection{Development of introgression lines (ILs)}

Fig. 1 shows the selection scheme for developing WTR1 ILs starting with the first round of selection of the WTR1/HAN//WTR1 $\mathrm{BC}_{1} \mathrm{~F}_{2}$ population with 576 plants under each of the IG, LI and RF conditions. In the first round selection, 46 plants were visually selected based on the desirable plant type and better yield performances than WTR1 (selection intensity, SI $=2.7 \%$ ) in 2011WS, including 17 plants from IG, 15 plants from LI, and 14 plants from RF. In the next season of 2012DS, the 46 BC1F3 lines (1,104 plants) were planted in single lines under the six different nutrient conditions, from which 71 plants were selected, including 7, 17, 13, 4, 19 and 13 plants from the NPK, -N, -P, -NP and -NPK conditions, respectively. In the following three consecutive seasons of 2012WS, 2013DS, and 2013WS, the same phenotypic selection was performed, resulting in $121 \mathrm{BC}_{1} \mathrm{~F}_{4}$ plants, $202 \mathrm{BC}_{1} \mathrm{~F}_{5}$ plants, and $230 \mathrm{BC}_{1} \mathrm{~F}_{6}$ plants, respectively. Taking together, the cumulated contribution of the four rounds of selection through progeny testing under the NPK, -N, -P, -NP and -NPK conditions leading to the final $230 \mathrm{BC} 1 \mathrm{F7}$ (ILs) was 0.107 for NPK, 0.144 for $75 \mathrm{~N}, 0.180$ for $-\mathrm{N}, 0.088$ for $-\mathrm{P}, 0.208$ for -NP and 0.273 for -NPK.

\subsection{Phenotypic performances of ILs for agro-morphological and yield traits in diverse environments}

In the replicated progeny testing, the recipient and donor had similar average grain yield across all six nutrient input conditions except under -NPK and -P. In the former case, WTR1 had significantly higher GY than the donor, HAN, while the opposite was true in the latter case under-P (Table 1). When the effects of different nutrient treatments were compared, the mean yields of the nutrient treatments could be divided into three levels: (1) the normal NPK showing the highest mean yield of the tested materials, and showed non-significant differences to $75 \mathrm{~N}$; (2) -P having the second highest average yield; and (3) -N, -NP, and -NPK showing the similarly low average GY for the tested lines. The nutrient treatments had similar effects on $\mathrm{PH}, \mathrm{TN}$, and biomass and had no effects on HD, TGW and SF (Fig. 2). However, the deficiency of nitrogen (-N) and phosphorus (-NP) caused significant 
reductions in SN, FNP, FLL, and FLA in comparison to the remaining five NuUE conditions. Importantly, the ILs showed similar mean values but tremendous variation and transgressive segregation for GY under all six nutrient conditions.

Table 1. The mean grain yield performances of 230 ILs and their parents under six nutrient input conditions

\begin{tabular}{lcccccc}
\hline \multirow{2}{*}{$\begin{array}{c}\text { NuUE } \\
\text { conditions }\end{array}$} & \multirow{2}{*}{ WTR1 } & HAN & Difference & \multicolumn{2}{c}{ ILs } & \multirow{2}{*}{\begin{tabular}{c} 
DMRT \\
\cline { 5 - 6 } comparison
\end{tabular}} \\
\hline -N & 21.0 & 21.6 & -0.6 & $13.9 \sim 35.0$ & $21.6 \pm 3.8$ & $\mathrm{~A}$ \\
-NP & 20.5 & 18.3 & 2.2 & $11.9 \sim 40.5$ & $20.5 \pm 4.5$ & $\mathrm{~A}$ \\
-NPK & 21.4 & 17.9 & $3.5^{*}$ & $12.8 \sim 43.0$ & $20.5 \pm 4.5$ & $\mathrm{~A}$ \\
-P & 25.9 & 28.4 & $-2.5^{*}$ & $17.6 \sim 42.2$ & $26.8 \pm 4.8$ & $\mathrm{~B}$ \\
$75 \mathrm{~N}$ & 29.1 & 30.1 & -1.0 & $20.8 \sim 48.8$ & $32.6 \pm 5.5$ & $\mathrm{C}$ \\
NPK & 32.8 & 31.9 & 0.9 & $19.3 \sim 50.3$ & $32.5 \pm 5.2$ & $\mathrm{C}$ \\
Mean & $25.1 \pm 5.1$ & $24.7 \pm 6.2$ & 0.4 & & & \\
\hline
\end{tabular}

DMRT=Duncan's multiple range tests for comparison of treatments; different letters and * indicate significant differences at $P \leq 0.05$, while the same letter indicates non-significant differences.

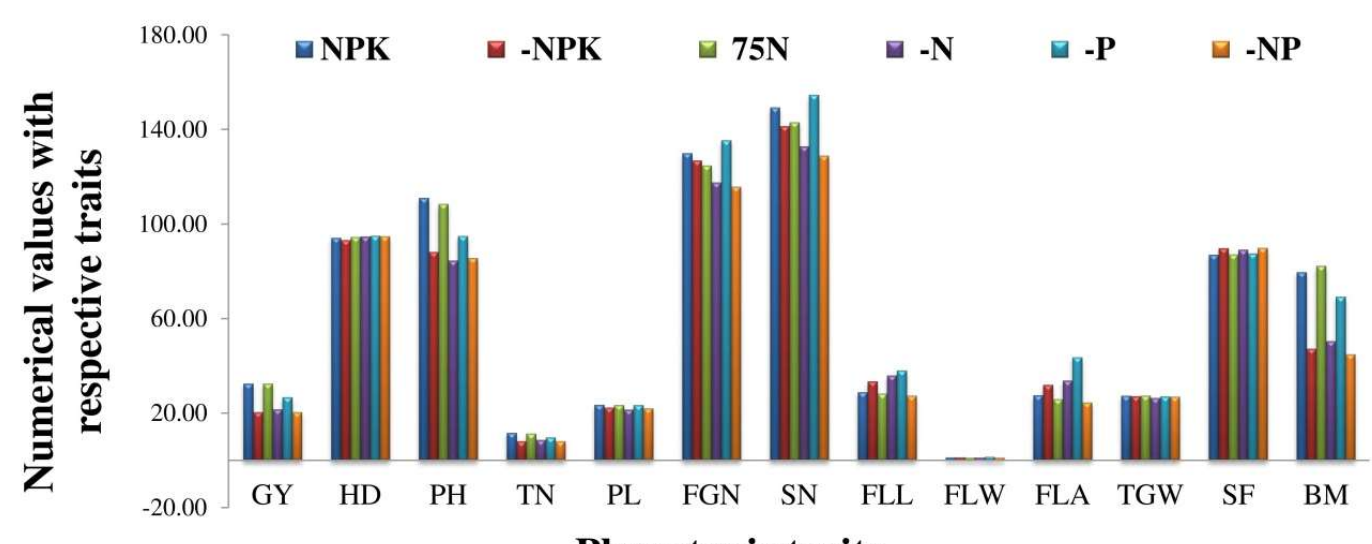

\section{Phenotypic traits}

Figure 2. Effects of six nutrient conditions on the average expressions of grain yield per plant (in $g$, GY) and related agro-morphological and yield attributed traits of 230 WTR1 introgression lines

GY=grain yield per plant(g); HD=heading date(days); $\mathbf{S N = s p i k e l e t ~ n u m b e r ~ p e r ~ p a n i c l e ; ~} \mathbf{G N = f i l l e d}$ grain number per panicle; SF=spikelet fertility(\%); TGW=thousand-grain weight(g); $\mathbf{P H = p l a n t}$ height(cm); TN=tiller number per plant; $\mathbf{P L}=$ panicle length $(\mathrm{cm}) ; \quad F L L=f l a g-l e a f ~ l e n g t h(\mathrm{~cm})$; FLW=flag-leaf width(cm); FLA=flag-leaf area $\left(\mathrm{cm}^{2}\right)$; BM biomass(g)

Table 2 shows the correlation coefficients between the mean yield performances and other agronomic traits measured under different nutrient conditions. High and positive correlations between grain yield and biomass were observed across all six nutrient conditions, particularly under the -N, -NP and -NPK, which was followed by FLW, FLA, and TN. Surprisingly, contributions of the two other direct yield components (FGN and TGW) to grain yield were, though positive, much less important, particularly under the less nutrient deficiency conditions (NPK and 75N). 
Table 2 Correlations of GY with other related traits of 230 WTR1 introgression lines under six nutrient conditions

\begin{tabular}{|c|c|c|c|c|c|c|c|c|c|c|c|c|}
\hline GY & HD & SN & FGN & $\mathrm{SF}$ & TGW & PH & TN & PL & FLL & FLW & FLA & BM \\
\hline$-\mathrm{N}$ & -0.12 & 0.03 & 0.10 & $0.18^{* *}$ & $0.17^{*}$ & $0.20^{* *}$ & $0.20^{* *}$ & $0.23^{* * *}$ & 0.09 & $0.19^{* *}$ & $0.18^{* *}$ & $0.35^{* * *}$ \\
\hline -NP & $-0.16^{*}$ & $0.31^{* * * *}$ & $0.31^{* * *}$ & 0.02 & 0.09 & $0.52^{* * * *}$ & $0.62^{* * *}$ & $0.39^{* * *}$ & $0.33^{* * *}$ & $0.52^{* * *}$ & $0.50^{* * *}$ & $0.74^{* * * *}$ \\
\hline -NPK & $-0.17^{*}$ & $0.21^{* *}$ & $0.27^{k * *}$ & $0.14^{*}$ & $0.24^{* * *}$ & $0.40^{* * *}$ & $0.60^{* * *}$ & $0.39^{* * *}$ & $0.41^{* * *}$ & $0.56^{* * *}$ & $0.57^{* * *}$ & $0.73^{* * * *}$ \\
\hline$-P$ & -0.04 & $0.19^{* *}$ & $0.20^{* *}$ & 0.11 & $0.36^{* * *}$ & 0.12 & $0.25^{* * *}$ & $0.24^{* * *}$ & -0.01 & 0.11 & 0.06 & $0.77^{* * * *}$ \\
\hline $75 \mathrm{~N}$ & -0.12 & $0.14^{*}$ & $0.15^{*}$ & 0.08 & 0.09 & 0.08 & $0.18^{* *}$ & $0.24^{* * *}$ & 0.09 & 0.07 & 0.11 & $0.26^{* * *}$ \\
\hline NPK & -0.09 & $0.18^{* *}$ & $0.22^{* * *}$ & $0.14^{*}$ & -0.01 & -0.05 & $0.16^{*}$ & $0.13^{*}$ & -0.08 & $-0.17^{* *}$ & $-0.16^{*}$ & $0.59^{* * * *}$ \\
\hline
\end{tabular}

The ILs showed tremendous segregation for their GY under different nutrient treatments (Table 2). In fact, a significant portion of ILs had significantly (10\%) higher GY than WTR1 under each of the nutrient treatments. In particular, more ILs showed significantly improved yields under the $75 \mathrm{~N}$ and -NP conditions, while the opposite was true under -P. Under the other conditions, the ILs showed largely normal distributions with approximately equal numbers of high and low yield ILs, providing tremendous opportunities for selection. Indeed, several promising ILs with significantly higher GY than WTR1 were identified under each of the NuUE treatments. These included Nue-114 (50.6 g), Nue-115 (42.9 g), Nue-3 (42.1 g), Nue-51 (42.1 g), Nue-112 (40.4 g) and Nue-230 (48.8 g) under the NPK, -NPK, -N, -P, -NP, and 75N conditions.

\subsection{Selection of promising ILs with superior yields under two or more nutrient conditions}

Table 3 shows the numbers of the selected 230 ILs from previous screening under the IG, LI and RF conditions that had significantly higher mean grain yields under one or two nutrient conditions. Of the 230 selected ILs, the number of ILs showing significantly higher GY than WTR1 was 52 under NPK, 103 under 75N, 33 under -P, 63 under -N, 52 under -NP, and 49 under -NPK, respectively. This translated into indirect selection efficiencies of $0.226,0.448,0.143,0.274,0.226$ and 0.213 for improved yield performances under the NPK, 75N, -P, -N, -NP and -NPK conditions, respectively. Of the 52 high yielding ILs identified under the normal NPK conditions, 26, 7, 17, 20 and 17 ILs also showed significantly improved GY under the 75N, -P, -N, -NP, and -NPK conditions, respectively. Of the 103 high yielding ILs selected under 75N, 20, 31, 34, 31 and 26 ILs showed significantly improved GY under the normal NPK, -P, -N, -NP, and -NPK conditions, respectively. Of the 33 high yielding, ILs identified under the -P condition, 20, 7, 13, 15 and 9 of these ILs performed well with significantly improved GY under the normal NPK, 75N, -N, -NP, and -NPK conditions, respectively. Similarly, of the 63 high yielding ILs selected under the -N condition, 13, 31, 17, 22 and 20 of these ILs showed significantly improved GY under the -P, normal NPK, 75N, -NP, and -NPK conditions, respectively. From the 52 high yielding ILs selected under the -NP condition, 22, 15, 34, 20, we were able to identify 20 ILs showing significantly improved GY under the -N, -P, normal NPK, 75N, and -NPK conditions, respectively. Finally, from the 49 high yielding ILs under -NPK, 20, 27, 9, 31 and 17 ILs also showed significantly improved yields under the NPK, 75N, -P, -N, and -NP conditions, respectively. This could be converted into an average secondary indirect selection efficiency of 0.335 for NPK, 0.276 for $75 \mathrm{~N}, 0.388$ for $-\mathrm{P}, 0.327$ for $-\mathrm{N}, 0.454$ for $-\mathrm{NP}$ and 0.424 for $-\mathrm{NPK}$, respectively. In other words, replicated progeny testing under the -NP and -NPK had resulted in the highest efficiency for selecting improved grain yields across all six nutrient conditions.

Table 3 Performances of WTR1 introgression lines (ILs) with 10\% (statistically significantly) higher and lower GY than the recipient

\begin{tabular}{ll}
\hline NuUE & $10 \%$ higher \\
\hline
\end{tabular}




\begin{tabular}{ccccccc}
\hline conditions & No. & Mean \pm SD & Range & No. & Mean \pm SD & Range \\
\hline NPK & 52 & $39.9 \pm 3.3$ & $36.1 \sim 50.3$ & 51 & $26.2 \pm 2.3$ & $19.3 \sim 28.7$ \\
$75 \mathrm{~N}$ & 103 & $37.7 \pm 3.2$ & $33.2 \sim 48.8$ & 23 & $23.9 \pm 1.6$ & $20.8 \sim 25.7$ \\
-P & 33 & $35.2 \pm 3.2$ & $31.4 \sim 42.2$ & 58 & $21.3 \pm 1.6$ & $17.6 \sim 23.3$ \\
-N & 63 & $26.5 \pm 2.7$ & $23.8 \sim 35.0$ & 57 & $17.4 \pm 1.2$ & $13.9 \sim 18.9$ \\
-NP & 52 & $26.8 \pm 4.3$ & $22.6 \sim 40.5$ & 28 & $14.7 \pm 1.2$ & $11.9 \sim 16.4$ \\
-NPK & 49 & $27.2 \pm 3.8$ & $23.6 \sim 43.0$ & 37 & $15.0 \pm 0.9$ & $12.8 \sim 16.1$ \\
\hline
\end{tabular}

Table 4 shows the numbers of low yielding ILs under different conditions from all high yielding ILs identified under each of the six nutrient conditions. Generally, a small portion of the ILs showing superior yields under one nutrient condition was yielding poorly under the other condition(s), suggested a low correlation for grain yield performances between different nutrient conditions.

Table 4 The numbers of WTR1 introgression lines showing significantly higher grain yields under two nutrient conditions

\begin{tabular}{lcccccc}
\hline $\begin{array}{l}\text { NuUE } \\
\text { conditions }\end{array}$ & NPK & $\mathbf{7 5 N}$ & $\mathbf{- P}$ & $\mathbf{- N}$ & -NP & -NPK \\
\hline NPK & 52 & 26 & 7 & 17 & 20 & 17 \\
$75 N$ & & 103 & 20 & 31 & 34 & 31 \\
-P & & & 33 & 13 & 15 & 9 \\
-N & & & 63 & 22 & 20 \\
-NP & & & & 52 & 27 \\
-NPK & & & & & & 49 \\
\hline
\end{tabular}

The values on the first diagonal show the numbers of higher GY ILs in each of the nutrient condition and values in the upper triangular show the numbers of higher GY ILs shared in each pair of the nutrient conditions.

\subsection{GY performances of groups divided by the type of first-round selection}

To examine how the types of the first round selection affected the selection efficiency for improving NuUE, the 230 ILs were divided into three groups, including 43 ILs originally selected from the IG conditions, 21 ILs from the LI conditions, and 166 ILs from the RF conditions. Surprisingly, ILs originally selected from the LI conditions showed the lowest mean GY under -NP, -NPK and -P, but highest mean GY under 75N and NPK, indicating that these ILs adapted better under good nutrient conditions, but very sensitive to P deficiency (Fig. 3). In contrast, those ILs originally selected from the IG conditions showed relatively better GY under -N and -NPK but performed poorly under $75 \mathrm{~N}$ and NPK, suggesting they were less sensitive to N deficiency, while those ILs originally selected from the RF conditions appeared to perform slightly better under the -P conditions. 

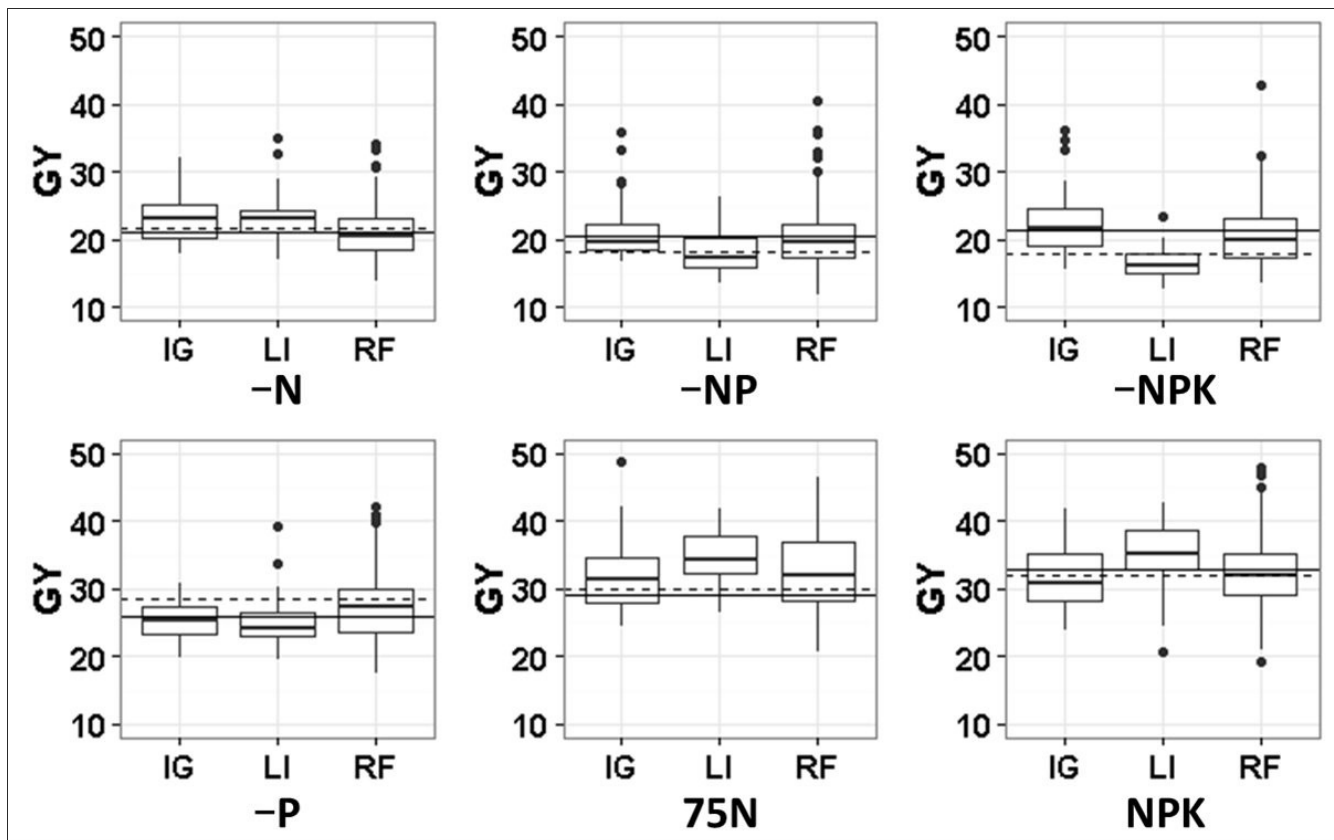

Figure 3. Comparison of mean GY performances between introgression line groups divided by the type of first-round selection, in which solid and broken lines indicate the values of the recipient, WTR1, and the donor, HAN, respectively. [IG = irrigated; LI = low input; $\mathbf{R F}=$ rainfed (drought)]

We indeed observed a negative correlation between yield performances under $-\mathrm{P}$ and $-\mathrm{N}$ conditions in some ILs. For example, five high yielding ILs (Nue-104, Nue-46, Nue-57, Nue-77, and Nue-83) identified under the -P conditions showed poor yield performances under the $-\mathrm{N}$ conditions (Table 6). When compared with WTR1, the poor yield performances under -N could be primarily attributed to their greatly reduced biomass, TN, and FGN.

Table 6. Grain yield performances of five WTR1 ILs and their parents with high GY in the -P condition but low GY in the -N condition

\begin{tabular}{lcccccccccccccc}
\hline \multirow{2}{*}{ Entry } & $\begin{array}{c}\text { NuUE } \\
\text { Con. }\end{array}$ & GY & HD & PH & TN & SN & FGN & SF & TGW & PL & FLL & FLW & FLA & BM \\
\hline WTR1 & & 25.9 & 91.5 & 96.7 & 13.2 & 199.6 & 158.3 & 87.6 & 24.9 & 24.2 & 41.2 & 1.5 & 46.8 & 94.7 \\
HAN & -P & 28.4 & 90.5 & 89.0 & 10.0 & 149.4 & 137.1 & 91.2 & 22.6 & 23.4 & 37.2 & 1.5 & 41.4 & 69.9 \\
\hline WTR1 & & 21.0 & 96.0 & 100.4 & 12.5 & 161.6 & 130.0 & 78.1 & 25.7 & 19.9 & 39.2 & 1.2 & 36.3 & 75.1 \\
HAN & $-\mathrm{N}$ & 21.6 & 87.0 & 81.6 & 13.5 & 112.4 & 106.9 & 96.6 & 27.1 & 21.7 & 38.2 & 1.3 & 37.7 & 128.7 \\
\hline Nue-104 & & 31.7 & 96.0 & 96.7 & 11.5 & 152.3 & 129.0 & 85.0 & 26.9 & 23.9 & 38.4 & 1.6 & 44.7 & 71.4 \\
Nue-46 & & 31.7 & 93.5 & 93.3 & 8.7 & 142.9 & 127.7 & 89.4 & 30.3 & 22.9 & 37.2 & 1.7 & 46.1 & 57.0 \\
Nue-57 & & 33.6 & 95.5 & 97.0 & 8.3 & 153.5 & 131.4 & 85.2 & 33.0 & 23.9 & 33.7 & 1.6 & 40.4 & 80.8 \\
Nue-77 & $-\mathrm{P}$ & 39.9 & 96.0 & 96.5 & 14.0 & 150.4 & 132.0 & 87.8 & 31.0 & 24.3 & 38.4 & 1.5 & 42.8 & 83.3 \\
Nue-83 & & 35.3 & 95.0 & 92.3 & 10.5 & 173.1 & 156.7 & 90.4 & 28.8 & 24.0 & 38.4 & 1.7 & 48.1 & 110.2 \\
Mean & & 34.4 & 95.2 & 95.2 & 10.6 & 154.4 & 135.4 & 87.5 & 30.0 & 23.8 & 37.2 & 1.6 & 44.4 & 80.5 \\
\hline Nue-104 & & 17.0 & 94.5 & 84.3 & 9.2 & 149.9 & 138.5 & 94.2 & 26.5 & 22.6 & 35.9 & 1.3 & 35.9 & 54.7 \\
Nue-46 & $-\mathrm{N}$ & 16.8 & 93.5 & 86.4 & 9.7 & 122.3 & 107.9 & 92.7 & 28.1 & 22.4 & 34.6 & 1.2 & 32.0 & 50.5
\end{tabular}




\begin{tabular}{llllllllllllll} 
Nue-57 & 18.1 & 93.5 & 79.4 & 12.2 & 126.6 & 92.4 & 81.3 & 28.7 & 21.8 & 36.8 & 1.3 & 36.4 & 39.9 \\
Nue-77 & 18.5 & 94.0 & 83.8 & 8.3 & 121.5 & 109.3 & 89.8 & 29.4 & 21.4 & 37.4 & 1.3 & 37.0 & 49.1 \\
Nue-83 & 16.6 & 96.5 & 82.6 & 8.3 & 102.5 & 88.6 & 89.8 & 25.1 & 20.2 & 33.0 & 1.2 & 29.3 & 40.3 \\
Mean & 17.4 & 94.4 & 83.3 & 9.5 & 124.6 & 107.4 & 89.6 & 27.5 & 21.7 & 35.6 & 1.3 & 34.1 & 46.9 \\
\hline
\end{tabular}

Con=conditions; GY=grain yield per plant(g); HD=heading date(days); $\mathbf{S N = s p i k e l e t ~ n u m b e r ~ p e r ~}$ panicle; FGN=filled grain number per panicle; SF=spikelet fertility(\%); TGW=thousand-grain weight(g); $\mathbf{P H = p l a n t ~ h e i g h t ( c m ) ; ~ T N = t i l l e r ~ n u m b e r ~ p e r ~ p l a n t ; ~} \mathbf{P L = p a n i c l e ~ l e n g t h ( c m ) ; ~ F L L = f l a g - l e a f ~}$ length(cm); FLW=flag-leaf width(cm); FLA=flag-leaf area $\left(\mathrm{cm}^{2}\right) ; \mathbf{B M = b i o m a s s}(\mathrm{g})$

\subsection{Promising ILs under different NuUE conditions}

Table 7 lists 12 ILs that showed significantly higher grain yields than WTR1 under two or more nutrient conditions and the same yields as WTR1 under the other conditions. These included one promising line, Nue-115, which had significantly improved yields under all four nutrient deficient conditions, four ILs (Nue-114, Nue-112, Nue-229, and Nue-230), that had superior yield performances under the normal or N75 plus two nutrient deficiency conditions. These lines have now promoted to the replicated multi-location yield trials for further testing for potential releases as new varieties in the target environments of several Asian and African countries.

Table 7 Twelve promising higher ILs with superior yield performances in two or more nutrient conditions

\begin{tabular}{|c|c|c|c|c|c|c|c|c|}
\hline \multirow[t]{2}{*}{ S. No } & \multirow[t]{2}{*}{ ILs } & \multirow[t]{2}{*}{ Designation } & \multicolumn{6}{|c|}{ NuUE Combinations } \\
\hline & & & NPK & $75 \mathrm{~N}$ & $-\mathbf{N}$ & $-\mathbf{N P}$ & -NPK & $-\mathbf{P}$ \\
\hline 1 & Nue-57 & GSR IR2-1-L1-NU1-NU1-NU1-NU1 & $\sqrt{ }$ & $\sqrt{ }$ & - & - & - & - \\
\hline 2 & Nue-60 & GSR IR2-1-RF6-NU3-NU4-NU68-NU35 & - & $\sqrt{ }$ & - & - & - & $\sqrt{ }$ \\
\hline 3 & Nue-77 & GSR IR2-1-RF6-NU3-NU4-NU7-NU38 & - & - & - & $\sqrt{ }$ & - & $\sqrt{ }$ \\
\hline 4 & Nue-86 & GSR IR2-1-RF6-NU4-NU7-NU60-NU56 & - & - & - & $\sqrt{ }$ & - & $\sqrt{ }$ \\
\hline 5 & Nue-106 & GSR IR2-1-RF6-NU4-NU9-NU14-NU66 & $\sqrt{ }$ & $\sqrt{ }$ & - & - & - & - \\
\hline 6 & Nue-118 & GSR IR2-1-RF6-NU6-NU1-NU20-NU88 & $\sqrt{ }$ & - & $\sqrt{ }$ & - & - & - \\
\hline 7 & Nue-228 & GSR IR2-1-RF6-NU7-NU2-NU77-NU94 & - & - & - & $\sqrt{ }$ & $\sqrt{ }$ & - \\
\hline 8 & Nue-112 & GSR IR2-1-RF6-NU7-NU2-NU76-NU96 & $\sqrt{ }$ & - & - & $\sqrt{ }$ & $\sqrt{ }$ & - \\
\hline 9 & Nue-114 & GSR IR2-1-RF6-NU7-NU3-NU82-NU97 & $\sqrt{ }$ & - & $\sqrt{ }$ & $\sqrt{ }$ & - & - \\
\hline 10 & Nue-229 & GSR IR2-1-RF6-NU7-NU2-NU37-NU100 & - & $\sqrt{ }$ & - & $\sqrt{ }$ & $\sqrt{ }$ & - \\
\hline 11 & Nue-230 & GSR IR2-1-Y17-NU2-NU5-NU6-NU7 & - & $\sqrt{ }$ & - & $\sqrt{ }$ & $\sqrt{ }$ & - \\
\hline 12 & Nue-115 & GSR IR2-1-Y17-NU2-NU5-NU6-NU8 & - & - & $\sqrt{ }$ & $\sqrt{ }$ & $\sqrt{ }$ & $\sqrt{ }$ \\
\hline
\end{tabular}

$\sqrt{ }$-Response to high GY in the specific nutrient condition; - No response to high GY

\section{Discussions}

Development and wide adoption of GSR cultivars that can produce high and stable yields under less inputs have been considered as an essential step to achieve sustainable agriculture and maintain environmental quality [10]. Thus, greatly improved NuUE is one of the important green target traits in breeding GSR cultivars. However, plant breeders are facing at least three great challenges for improving NuUE. In the first place, improved NuUE is at most the secondary target trait in virtually all breeding programs because improved yield potential and adaptation to key major abiotic stresses in the target ecosystems would always be the number one priority in most breeding programs. For 
example, adequate tolerance to drought and salinity, in addition to the high yield potential, would be the priority traits for breeding programs for the rainfed ecosystem and saline areas. The second difficulty comes from the fact that NuUE is a complex trait of low heritability, which is affected most by the intrinsic large variation in soil fertility and type. The third challenge is apparent 'lack' of appropriate high NuUE donors because of fewer past efforts to identify rice germplasm accessions with high NuUE. As a result, there have been few well-documented breeding efforts for developing rice cultivars tolerant to nitrogen and phosphorus deficiencies [21, 22, 23, 24]. In this respect, results from this study provided at least partial answers to the three practical questions and demonstrated an innovative breeding method involving four consecutive rounds of selections under six different NuUE conditions for developing rice varieties with significantly improved NuUE.

\subsection{Exploiting the 'hidden' genetic diversity in different sub-specific gene pools for improving NuUE}

In the BC breeding procedure for developing ILs with improved NuUE, we used HAN, a Geng variety as the donor, to improve NuUE of a widely adaptable Xian variety, WTR1. WTR1 was released in Bangladesh as BRRI dhan69 in 2015 under the Green Super Rice (GSR) project for the 'Boro' conditions after six years of rigorous testing for grain yield and grain quality. Our results indicated that HAN did not seem to have a high NuUE but was able to contribute many useful genes/traits into WTR1, resulting in the development of many WTR1 ILs with greatly improved NuUE. This was consistent with our previous results that there is rich hidden genetic diversity in the different sub-specific gene pools that can be exploited for improving complex traits such as abiotic/biotic stress tolerances/resistances [23, 25, 26, 27,] using a modified BC breeding strategy [ 10, 23, 28 ]. This is not surprising because it is now known that different rice populations, particularly the two major subspecies, each contains large numbers of unique genes and alleles (haplotypes) that are absent or rare in other populations or subspecies ([19]. Thus, results from this study indicated that accessions of different subspecies could be a good source of useful genetic diversity for improving NuUE in rice.

\subsection{Promising lines with high NuUE and selection efficiency for improved NuUE}

In this study, we were able to identify many ILs, out of the $230 \mathrm{ILs}$, that had significantly higher yields (>30g in GY) than WTR1 under at least one nutrient deficiency condition, including 10 ILs under -NPK, 7 ILs under -N, 47 ILs under -P, 9 ILs under -NP, plus 12 promising ILs with significantly improved NuUE under more than one nutrient deficient conditions (Table 7). While this is consistent with other findings $[27,28]$ that high yielding varieties with high NuUE can be developed under NuUE deficiency conditions, it would be interesting to understand how the various types of selection schemes we adopted affect our selection efficiency for improving NuUE in rice. For the first round selection, we had approximately the same selection intensity under the IG, LI, and RF conditions, of which LI and RF were relevant to NuUE. Under the LI condition, we expected to select those plants which were tolerant to low NPK, because zero nutrient input was given to the LI field for 16 consecutive crop seasons before the selection. Surprisingly, the ILs selected under LI did not have improved yield under the -NP, -NPK, and -P conditions. Instead, these ILs had higher grain yields under the $-\mathrm{N}, 75 \mathrm{~N}$, and NPK conditions, when compared with those selected from IG and RF (Fig. 3). According to the soil fertility analyses, the available soil NPK contents of the LI field has reached a fairly constant low levels at $0.23 \mathrm{~N}[\mathrm{KJn}(\%)], 24 \mathrm{P}-\mathrm{Bray}(\mathrm{mg} / \mathrm{kg})$ and $1.19 \mathrm{meq} / 100 \mathrm{~g}$ ) in an average of both the seasons of 2012 (WS and DS), 2013 (WS and DS), and 2014 (DS). Taking together, our results seemed to suggest these lines selected under LI had low P uptake ability but high $\mathrm{P}$ usability, and high $\mathrm{N}$ uptake and usability, which remain to be validated by more accurately, designed experiments. On the other hand, water deficiency was the primary factor liming rice yield performance under the RF condition where the first round selection was practiced. It was reported that plant water use efficiency is closely related to their NuUE because water uptake would facilitate nutrient uptake [24]. However, those ILs selected under RF showed relatively better GY under the $-\mathrm{P}$ and $75 \mathrm{~N}$ condition and a much greater variation (72.1\%) in GY and 
all measured traits across the six nutrient conditions than those selected from either IG or LI (Fig. 3). Furthermore, the IL (Nue-112) with highest NuUE was also selected from the RF conditions (Table 7). It suggested that drought tolerance and high NuUE in this population were apparently under independent genetic control. However, it is important to test if this is held generally true for other rice populations. For those ILs originally selected under IG (irrigated conditions), no overall yield advantages under the nutrient deficiency conditions were observed. Overall, the efficiency of the first round selection was RF > LI > IG for improving NuUE. Therefore, selecting of NuUE rice cultivars under rainfed situation is a prominent approach for the identification of superior rice cultivars.

\subsection{Traits contributed to NuUE of rice}

Our results indicated that high NuUE in rice is a very complex trait controlled by different genetic, physiological and molecular mechanisms. This conclusion was implicated by at least two observations. Firstly, the contributions of the three yield components to GY were relatively weak and varied considerably with each nutrient deficiency condition, though high and positive correlations between GY and BM were consistently observed across all nutrient deficient conditions. This was in contrast to previous reports that higher yields from enhanced grain filling ratio (GFR), spikelet number per panicle (SNP), number of panicles (NP) and TGW under nutrient deficiency conditions could be attributed to better nutrient absorption and uptake by plants after the application of fertilizer sources [29-34]. Earlier reports also indicated that grain number and weight primarily controlled by $\mathrm{P}$ and $\mathrm{N}[35,36,37]$, because $\mathrm{P}$ is more utilized in the formation of grain productivity and $\mathrm{N}$ influences tillering ability which contributed to higher yields through more panicles [38, 39]. Indeed, we observed that of the six nutrient conditions, NPK produced the highest $\mathrm{PH}$, and the lowest $\mathrm{PH}$ was recorded in the $-\mathrm{N}$ condition. In the case of -NP condition, FLL, FLW and FLA traits were less and higher in the -P condition. TN was high in NPK, and low in 75N. Similarly, higher TN leading higher GY was also reported in rice [40]. Apparently, increased GY could be attributed to possible higher photo-assimilates and dry matter accumulation from increased leaf area in response to $\mathrm{N}$ fertilizers [41, 42]. TN, LL, LW, and LA were also reportedly significantly improved by the mining of nutrients, particularly $\mathrm{N}$, through better root development, leading to improved translocation of carbohydrate capacity from expanded leaf rate [42, 43, 44]. Secondly, we observed that some ILs showed higher GY under one nutrient condition but lower GY in another (Table 5), while high GY of different ILs could be attributed to different combinations of yield components. For example, the high GY of Nue-112 under the NPK condition was mainly attributed to higher FGN, SN, PL, BM but not TGW because the TGW of Nue-112 was significantly lower than WTR1. However, its high GY under other conditions could be attributed to the simultaneous improvement of FGN, PL, BM, and TGW or the balance between them. Similarly, the high GY of Nue-115 could primarily be attributed to TGW, FLW (-NPK), FLW (-P), TGW (-N), respectively. Thus, the 230 ILs and their tremendous variation in NuUE provide useful materials for studying the genetic and physiological mechanisms of NuUE of rice, which is in progress.

\section{Conclusions}

Breeding of improved NuUE rice varieties is important for sustaining the rice production with depleting nutrient resources in the coming decades [45]. NuUE is a complex trait of low heritability, which is affected most by the intrinsic large variation in soil fertility and type [45]. Our study involved screening of inter-subspecific $\mathrm{BC}_{1} \mathrm{~F}_{2}$ population with first round under $\mathrm{RF}$, LI and IG and followed by a systematic phenotypic selection across six different nutrient conditions over four rounds that helped us to identify improved NuUE introgression lines. The study revealed that HAN did not possess a high NuUE but was able to contribute many useful genes/traits into WTR1, resulting in the development of many WTR1 ILs with greatly improved NuUE. These results confirm that there is rich hidden genetic diversity in the different sub-specific gene pools that can be exploited for improving complex traits such as abiotic/biotic stress tolerances/resistances using a modified BC breeding strategy. However, through this study, we could develop as many 230 ILs 
that is being utilized for studying the genetic and physiological mechanisms of NuUE of rice for different target NuUE related traits. The study demonstrated an efficient inter-subspecific BC breeding procedure with first round selection under the rainfed-drought condition followed by four generations of progeny testing for yield performances under six different nutrient conditions. Overall, the efficiency of the first round selection was RF > LI > IG for improving NuUE. Therefore, selecting of NuUE rice cultivars under rainfed situation is a prominent approach for the identification of superior rice cultivars. Identification of several promising introgression lines (Nue-115, Nue-114, Nue-112, Nue-229, and Nue-230) with improved NuUE would help rice breeding programs to utilize these materials for initiating new crosses and develop new breeding materials. These lines have now been promoted to the replicated multi-location yield trials for further testing for potential releases as new varieties in the target environments of several Asian and African countries.

Author Contributions: Z.A.J., J.A., and A.M. worked on outlining the contents of the manuscript and prepared the draft article. J.A., J.H., J.X., and Z.L. contributed to the screening methodologies. Z.A.J., Y.P., B.A., and A.M., curated the data and analyzed. J.A. and Z.L. conceived of the basic idea, gave suggestions, corrected the entire article and improved the prospects for breeding programs. All the authors read and approved the review article.

Acknowledgments: The authors would like to thank and acknowledge the Bill \& Melinda Gates Foundation (BMGF) for providing a research grant to Z.L. for the Green Super Rice project under ID OPP1130530. We would also like to thank the Department of Agriculture (DA) of the Philippines for providing funds to J.A. under the Next-Gen project.

Conflicts of Interest: The authors declare that the research review was conducted in the absence of any commercial or economic associations that could be construed as a potential conflict of interest. 


\section{References}

1. Redona, E.D. Rice biotechnology for developing countries in Asia, Agric. Biotechnol, Philippine Rice Research Institute Science, Philippines. 2004, pp. 201-230.

2. Pingali, P.L. Green Revolution: impacts, limits, and the path ahead. Proc. Natl. Acad. Sci. U.S.A. 2012, 109, 12302-12308.

3. Zhang, Q. Strategies for developing Green Super Rice. Proc. Natl. Acad. Sci. U.S.A. 2007, 104, 16402-16409.

4. Cassman, K.G.; Peng, S.; Olk, D.C.; Ladha, J.K.; Reichardt, W.; Dobermann, A.; Singh, U. Opportunities for increased nitrogen-use efficiency from improved resource management in irrigated rice systems. Field Crops Res. 1998, 56, 7-39.

5. Senthilvel, S.; Govindaraj, P.; Arumugachamy, A.; Latha, R.; Malarvizhi, P.; Gopalan, A.; Maheswaran, M. Mapping genetic loci associated with nitrogen use efficiency in rice (Oryza sativa L.), In Proceeding of New directions for a diverse planet: Proceedings of the $4^{\text {th }}$ International Crop Science Congress, Brisbane, Australia, 2004.

6. Ladha, J.K.; Pathak, H.; Krupnik, T.J.; Six, J.; van Kessel, C. Efficiency of fertilizer nitrogen in cereal production: retrospects and prospects. Adv. Agron. 2005, 87, 85-156.

7. Roberts, T.L. Improving nutrient use efficiency, Turk. J. Agric. For. 2008, 32, 177-182.

8. Fageria, N.K.; Baligar, V.C. Enhancing nitrogen use efficiency in crop plants. Adv. Agron. 2005, 88, 97-185.

9. Papademetriou, M.K. Rice production in the Asia-Pacific region: issues and perspectives. Bridging the Rice Yield Gap in the Asia-Pacific Region, Bangkok, Thailand, 2000, p. 220.

10. Ali, J.; Xu, J.L.; Gao, Y.M.; Ma, X.F.; Meng, L.J.; Wang, Y.; et al. Harnessing the hidden genetic diversity for improving multiple abiotic stress tolerance in rice (Oryza sativa L.). PLoS ONE. 2017, 12(3): e0172515.

11. Fageria, N.K.; Baligar, V.C.; Heinemann, A.B.; Carvalho, M.C.S. Nitrogen uptake and use efficiency in rice, In: A. Rakshit, B. H. Singh, and A. Sen, (eds.) Nutrient Use Efficiency: from Basics to Advances, Springer India, New Delhi, 2015, pp. 285-296.

12. Vinod, K.K.; Heuer, S. Approaches towards nitrogen- and phosphorus-efficient rice. AoB Plants. 2012, pls028.

13. Baligar, V.C.; Fageria, N.K.; He, Z.L. Nutrient use efficiency in plants, Commun. Soil Sci. Plant Anal. 2001, 32, 921-950.

14. Van Hach, C.; Nam, N. Responses of some promising high-yielding rice varieties to nitrogen fertilizer, Omon rice. 2006, 14, 78-91.

15. Saito, K.; Linquist, B.; Atlin, G.N.; Phanthaboon, K.; Shiraiwa, T.; Horie, T. Response of traditional and improved upland rice cultivars to $\mathrm{N}$ and $\mathrm{P}$ fertilizer in northern Laos, Field Crops Res. 2006, 96, 216-223.

16. Alam, M.; Ali, M.; Amin, A.; Hasanuzzaman, M. Yield attributes, yield and harvest index of three irrigated rice varieties under different levels of phosphorus, Adv. Biol. Res. 2009, 3, 132-139.

17. Abou-Khalifa, A.A.B. Evaluation of some rice varieties under different nitrogen levels, Adv. Appl. Sci. Res. 2012, 3, 1144-1149.

18. Sharma, R.; Gangwar, R.K.; Yadav, V.; Kumar, R. Response of basmati rice (Oryza sativa) cultivars to graded nitrogen levels under transplanted condition, Int. J. Res. Applied Nat. Social Sci. 2014, 2, 33-38. 
19. Wang, W.; Mauleon, R.; Hu, Z.; Chebotarov, D.; Tai, S.; Wu, Z.; Li, M.; Zheng, T.; Fuentes, R.R.; Zhang, F.; et al. Genomic variation in 3,010 diverse accessions of Asian cultivated rice. Nature. 2018, 557(7703), 43-49.

20. Core Team, R.R. A language and environment for statistical computing. R Foundation for Statistical Computing, Vienna, Austria, 2013, URL http://www.R-project.org/.

21. Panigrahy, M.; Rao, D.N.; Yugandhar, P.; Raju, N.S.; Krishnamurthya, P.; Voletia, S.R.; Reddy, G.A.; Mohapatra, T.; Robin, S.; Singh, A.K.; Singh, K.; Sheshshayeef, M.; Sharma, R.P.; Sarla, N. Hydroponic experiment for identification of tolerance traits developed by rice Nagina22 mutants to low-phosphorus in field condition, Arch. Agron. Soil. Sci. 2014, 60, 565-576

22. Fageria, N.K.; Oliveira, J.P. Nitrogen, Phosphorus and Potassium Interactions in Upland Rice, J Plant Nutrition. 2014, 37, 1586-1600.

23. Aluwihare, Y., Ishan, M.; Chamikara, M.; Weebadde, C.; Sirisena, D.; Samarasinghe, W.; Sooriyapathirana, S. Characterization and selection of phosphorus deficiency tolerant rice genotypes in Sri Lanka, Rice Sci. 2016, 23, 184-195.

24. Mahender, A.; Anandan, A.; Pradhan, S.K.; Singh, O.N. Traits-related QTLs and genes and their potential applications in rice improvement under low phosphorus condition, Arch. Agron. Soil Sci. 2017, 15, 1-16.

25. Ali, A.J.; Xu, J.L.; Ismail, A.M.; Fu, B. Y.; Vijaykumar, C.H.M.; Gao, Y.M. et al. Hidden diversity for abiotic and biotic stress tolerances in the primary gene pool of rice revealed by a large backcross breeding program. Field Crops Res. 2006, 97, 66-76.

26. He, Y.; Zheng, T.; Hao, X.; Wang, L.; Gao, Y.; Hua, Z.; et al. Yield performances of japonica introgression lines selected for drought tolerance in a BC breeding programme. Plant Breed. 2010, 129, 167-175.

27. Cui, Y.; Zhang, W.; Lin, X.; Xu, S.; Xu, J.; Li, Z. Simultaneous Improvement and Genetic Dissection of Drought Tolerance Using Selected Breeding Populations of Rice. Front Plant Sci. 2018, 9, 320. doi: 10.3389/fpls.2018.00320

28. Li, Z.; Fu, B.; Gao, Y.; Xu, J.; Ali, J.; Lafitte, H.; et al. Genomewide introgression lines and their use in genetic and molecular dissection of complex phenotypes in rice (Oryza sativa L.). Plant Mol. Biol. 2005, 59, 33-52.

29. Ali, J.; Xu, J.; Gao, Y.; Fontanilla, M.; Li, Z. Breeding for yield potential and enhanced productivity across different rice ecologies through Green Super Rice (GSR) breeding strategy, International Dialogue on Perception and Prospects of Designer Rice. Society for the Advancement of Rice Research, Directorate of Rice Research, Hyderabad, India, 2013, pp. 60-68.

30. Sarkar, B.; Naidu, R. Nutrient and water use efficiency in soil: the influence of geological mineral amendments, In: A. Rakshit, B. H. Singh, and A. Sen, (eds.) Nutrient Use Efficiency: from Basics to Advances, Springer India, New Delhi, 2015, pp. 29-44.

31. Ahmad, R.M.; Naveed, M.; Aslam, Z.A.; Arshad, M. Economizing the use of nitrogen fertilizer in wheat production through enriched compost, Rev. Agric. Food Sys. 2008, 23, 243-249. 
32. El-Refaee, I.S.; Ebaid, R.A.; El-Rewiny, I.M. Performance of rice (Oryza sativa L.) plant under different water regimes and methods of planting, Alex. J. Agric. Res. 2006, 51, 47-55.

33. Hossaen, M.A.; Shamsuddoha, A.T.M.; Paul, A.K.; Bhuiyan, M.S.I.; Zobaer, A.S.M. Efficacy of different organic manures and inorganic fertilizer on the yield and yield attributes of Boro rice, The Agriculturists. 2011, 9, 117-125.

34. Iqbal, A.S.; Abbasi, M.K.; Rasool, G. Integrated plant nutrition system (IPNS) in wheat under rainfed condition of Rawalkot Azad Jammu and Kashmir, Pak. J. Soil. Sci. 2002, 21, 79-86.

35. Ju, J.; Yamamoto, Y.; Wang, Y.L.; Shan, Y.H.; Dong, G.C.; Yoshida, T.; Miyazaki, A. Genotypic differences in grain yield, and nitrogen absorption and utilization in recombinant inbred lines of rice under hydroponic culture, Soil Sci. Plant Nutri. 2006, 52, 321-330.

36. Tong, H.; Chen, H.L.; Li, W.P.; Mei, H.W.; Xing, Y.Z.; Yu, X.Q.; Xu, X.Y.; Zhang, S.Q.; Luo, L.J. Identification and characterization of quantitative trait loci for grain yield and its components under different nitrogen fertilization levels in rice (Oryza sativa L.), Mol Breed. 2011, 28, 495-509.

37. Makino, A. Photosynthesis, grain yield, and nitrogen utilization in rice and wheat, Plant Physiol. 2011, 155, 125-129.

38. Yosef Tabar, S. Effect of nitrogen and phosphorus fertilizer on growth and yield rice (Oryza Sativa L), Int. J. Agron. Plant Prod. 2012, 3, 579-584.

39. Amanullah, Inamullah. Dry matter partitioning and harvest index differ in rice genotypes with variable rates of phosphorus and zinc nutrition, Rice Sci. 2016, 23, 78-87.

40. Lin, X.; Zhou, Q.W.J.; Zhu, D.F.; Zhang, Y.P. Effect of SWD irrigation on photosynthesis and grain yield of rice (Oryza sativa L.), Field Crops Res.2005, 94, 67-75.

41. Chaturvedi, I. Effect of Nitrogen Fertilizers on Growth, Yield and Quality of Hybrid Rice (Oryza sativa L.), J. Central Eur. Agric. 2005, 6, 611-618.

42. Mandal, N.N.; Chaudhry, P.P.; Sinha, D. Nitrogen, phosphorus and potash uptake of wheat (var. Sonalika), Env. and Eco. 1992, 10, 297.

43. Singh, S.; Ram, L.C. Effect of fertilizer and organic manure on root CEC of some rice and wheat varieties, J. Indian Soc. Soil Sci. 1976, 24, 427-31

44. Yoshida, S.; Cock, J.H.; Parao, F.T. Physiological aspects of high yield. Int. Rice Res. Inst. Rice breeding. 1972, pp. 455-469.

45. Ali, J.; Jewel, Z.A.; Mahender, A.; Anandan, A.; Hernandez, J.;Li Z. Molecular genetics and breeding for nutrient use efficiency in rice. Int. J. Mol. Sci. 2018, 19, 1762. 